\title{
Inquérito sorológico da infecção pelo Echinococcus sp no município de Sena Madureira, AC
}

\author{
A serological survey of the infection by Echinococcus sp. \\ in the municipal ity of Sena Madureira, AC
}

Ricardo Pastore' ${ }^{\text {, Lúcia H. Vitali², Vanize de Oliveira Macedo }{ }^{1} \text { e Aluízio Prata }}{ }^{3}$

\begin{abstract}
Resumo Foi realizado no município de Sena Madureira, Acre, um inquérito soroepidemiológico para avaliar o contato de indivíduos com o Echinococcus sp. Escolheu-se duas populações distintas, uma residente na área urbana e a outra na área rural do município, distribuída em comunidades ribeirinhas da região. Foram avaliados no total 1.064 indivíduos, dos quais 851 pertencentes à área urbana e 213 à área rural. O estudo foi dividido em duas partes: inquérito sorológico, no qual foram coletadas e enviadas amostras de sangue ao Laboratório de Sorologia do Hospital das Clínicas da Faculdade de Medicina de Ribeirão Preto da Universidade de São Paulo, onde foram realizados os testes imunológicos pela técnica de contraimunoeletroforese; inquérito epidemiológico para avaliação individual, além de as condições de moradia e hábitos dos indivíduos. A prevalência sorológica na área rural foi $6 \%$, enquanto na urbana fomos 3,5\%. A prevalência geral foi $4 \%$. Quando analisada a possibilidade da existência de outros hospedeiros intermediários no ciclo do Echinococcus vogeli, foram encontrados resultados que sugerem ser o porco doméstico o mais provável.
\end{abstract}

Palavras-chaves: Hidatidose policística. Hidatidose. Echinococcus vogeli. Amazônia brasileira.

Abstract A serological inquiry was performed in the municipality of Sena Madureira, Acre State, Brazil, to evaluate the individual contact with Echinococcus sp. The participants were recruited from two distinct populations: residents in the urban and rural areas, the latter distributed among riverside communities of the region. A total of 1,064 individuals were evaluated: 851 from the urban zone and 213 from the rural area. The study was divided into two phases: a serological screening, in which the blood samples were collected and then sent to the Hospital das Clínicas da Faculdade de Medicina de Ribeirão Preto, Universidade de São Paulo (Serology Laboratory), Ribeirão Preto, SP, Brazil, for the serological test by counterimmunoelectrophoresis technique; and secondly an epidemiological inquiry for evaluating the individuals and their dwelling conditions and customs. Comparing the results of serological tests, the prevalence in the rural area was $6 \%$ against $3.5 \%$ in the urban area. The overall prevalence was $4 \%$. The possibility of the existence of another intermediate host in the life cycle of Echinococcus vogeli was analyzed and the findings indicated the domestic pig as being the most probable.

Key-words: Polycystic hydatid disease. Hydatidosis. Echinococcus vogeli. Brazilian Amazonian Region.

Durante grande parte do século passado, o Echinococcus granulosus foi considerado o único agente da hidatidose na América do Sul, embora muitos casos atípicos tivessem sido descritos ${ }^{16101924}$. Em 1972, Rausch e Bernstein, descreveram uma nova espécie, 0 Echinococcus vogeli, que juntamente com o Echinococcus oligarthrus são considerados agentes da hidatidose policística (HP) ${ }^{28} 2931$.

A definição da paca (Cuniculus paca) como hospedeiro intermediário do ciclo biológico do E. vogeli foi dada por D'Alessandro e $\operatorname{cols}^{8}{ }^{9}$ que baseou-se na observação de animais silvestres da Amazônia Colombiana de 1962 a 1979. Esta conclusão é sustentada pela observação epidemiológica do contato entre doentes de hidatidose policística com cães domésticos alimentados a partir das vísceras desses animais ${ }^{1323}$.

Em publicação de 1997, D'Alessandro agrupou os casos de hidatidose policística até então conhecidos, fazendo uma metanálise referente ao período de 1979 a 1997. Foram relatados 72 casos em 11 diferentes

\footnotetext{
1. Núcleo de Medicina Tropical da Universidade de Brasília, Brasília, DF. 2. Laboratório de Sorologia do Hospital das Clínicas da Faculdade de Medicina da Universidade de São Paulo, Ribeirão Preto, SP. 3. Disciplina de Doenças Infecciosas e Parasitárias da Faculdade de Medicina do Triângulo Mineiro, Uberaba, MG. Endereço para correspondência: Dr Ricardo Pastore. Núcleo de Medicina Tropical/UnB. Caixa Postal 4517, 70919-970 Brasília, DF. Tel: 5561 2735008; Fax: 55612732811 e-mail: tropical@unb.br

Recebido para publicação em 8/2/2003

Aceito em 21/5/2003
} 
países da América Latina. O Brasil foi o país que apresentou o maior número com 24 casos relatados. Em adendo a esta publicação D'Alessandro cita o relato de 14 casos no estado do Pará que ainda não haviam sido publicados. Estes novos relatos elevam o número de casos brasileiros para 38 , que somados aos recém publicados ${ }^{2} 32$ perfazem 40.

Os indivíduos que apresentaram a HP têm características epidemiológicas comuns, são na sua maioria provenientes de áreas silvestres, relatando contato com cães domésticos alimentados com carne de paca ou cutia ${ }^{21} 24$. Em muitas localidades do Brasil, principalmente na Amazônia, a prática da caça de subsistência é comum, chegando a ser uma das principais fontes protéicas da dieta diária.

As manifestações clínicas da hidatidose policística demoram a surgir e durante o curso natural da infecção a evolução dos cistos é variável. Estas manifestações dependem do órgão afetado. O fígado é o mais comumente envolvido. As alterações abdominais mais comuns são, a dor e a ocorrência de massa palpável no epigástrio e hipocôndrio direito, resultante em sua maioria da hepatomegalia ou esplenomegalia (mais rara). Outras ocorrências comuns são o emagrecimento e a febre.

Vários métodos têm contribuído de forma decisiva para o estabelecimento precoce do diagnóstico. Desde os métodos de imagem (ultrassonografia (US) ${ }^{25}{ }^{26} \mathrm{e}$ tomografia computadorizada $(\mathrm{TC})^{16}$ até os métodos imunoenzimáticos. As técnicas mais empregadas são: contraimunoeletroforese, aglutinação com látex, (Enzymelinked immunosorbent assay) ELISA e mais recentemente o Immunoblot ${ }^{14151718}$. A contraimunoeletroforese (CIE) é uma técnica de imunodiagnóstico considerada de alta especificidade e relativa sensibilidade no diagnóstico das hidatidoses.

Não se pode precisar o tempo de evolução da doença e nem mesmo relacionar quantos daqueles infectados pelo Echinococcus vogeli ou oligarthrus evoluem como enfermos. As investigações populacionais podem trazer à luz estas informações.

Neste estudo o município escolhido foi o de Sena Madureira (Acre), onde descreveu-se a presença de cistos de Echinococcus vogeli em fígado de paca ${ }^{23}$. 0 Acre é o estado brasileiro onde foram relatados oito dos 25 casos de HP.

\section{POPULAÇÃO E MÉTODOS}

A área de estudo escolhida foi o município de Sena Madureira no Acre onde vive uma população de 29.412 pessoas, 16.148 (54,9\%) na área urbana, (Censo 2000, IBGE). E banhado pelo rio laco, que tem como seus principais afluentes os rios Caeté e Macauã).

Foram estudadas duas populações. A primeira constituída por uma amostra sistemática de moradores de um mesmo bairro (área urbana), e a segunda (área rural) constituída por uma amostragem em conglomerados de sete comunidades ribeirinhas banhadas pelos rios da região. $A$ abordagem dos indivíduos foi realizada através de entrevista e coleta de amostra de sangue para reação sorológica por contraimunoeletroforese.

Elaborou-se uma ficha para coleta de informações individuais (identificação, antecedentes pessoais e hábitos) e um questionário, onde foram aplicadas questões sobre as condições de moradia, presença de animais domésticos e hábitos familiares.
Para o inquérito sorológico foi padronizada a coleta de $10 \mathrm{ml}$ de sangue, em veia periférica, através de aparelho de vacutainer $\AA$, com técnica asséptica. O soro obtido pela centrifugação foi colocado em tubos tipo eppendorfs $\AA$ de $2 \mathrm{ml}$ e armazenado a $-20^{\circ} \mathrm{C}$ até o envio ao laboratório de sorologia do Hospital das Clínicas da Faculdade de Medicina de Ribeirão Preto da Universidade de São Paulo.

A escolha da contraimunoeletroforese (CIE) para detecção de anticorpos anti-equinococos foi baseada na disponibilidade do método e na experiência da equipe com casos de hidatidose policística. Para estabelecer critérios de comparação, coletouse e testou-se uma amostra de sangue de 46 indivíduos moradores da região de Ribeirão Preto, com resultados sorológicos negativos para triagem padrão em Banco de Sangue (HTLV, HIV, hepatite B e C, doença de Chagas e sífilis). Obteve-se 2 indivíduos com reação sorológica positiva e títulos 1/2. Passou-se a considerar os indivíduos com sorologia positiva e títulos de 1/2 ou abaixo como negativos.

\section{RESULTADOS}

Avaliou-se 1.064 indivíduos sendo 851 (80\%) procedente da área urbana do município e 213 da rural (Tabela 1). A positividade sorológica foi de $4 \%$, sendo

Tabela 1 - Procedência da população avaliada em Sena Madureira. Acre, 2001.

\begin{tabular}{lcc}
\hline População & Número & Porcentagem \\
\hline Urbana & 851 & 80,0 \\
Rural & 213 & 20,0 \\
\hline Total & 1.064 & 100,0 \\
\hline
\end{tabular}

$3,5 \%$ na urbana e $6 \%$ na rural (Tabela 2 ). Entre os 41 soropositivos, $32(78,5 \%)$ o foram com título de $1 /$ $4,8(19,5 \%)$ com $1 / 8$ e $1(2,4 \%)$ a $1 / 16$ (Tabela 3$)$.

Tabela 2 - Positividade da reação de contraimunoeletroforese para hidatidose verificada nas populacões rural e urbana avaliadas em Sena Madureira. Acre, 2001.

\begin{tabular}{lrrc}
\hline & \multicolumn{3}{c}{ População examinada } \\
\cline { 2 - 4 } & $\mathrm{n}^{\circ}$ & positivo & Intervalo de confiança $\alpha=5 \%$ \\
\hline Uural & 213 & $12(6 \%)$ & $3,26-10,44$ \\
\hline Total & 29 & $29(3,5 \%)$ & $2,41-5,09$ \\
\hline
\end{tabular}


No município de Sena Madureira, é grande o número de indivíduos que utilizam a carne de caça como alimento (cidade 89\% e área rural 99,1\%) (Tabela 4).

Um número elevado de indivíduos, 67,5\% (área urbana) e $68,7 \%$ (área rural) que utilizam a carne de animais silvestres como alimento, refere já ter consumido, pelo menos uma vez, a carne de paca.

\begin{tabular}{|c|c|c|c|c|}
\hline \multirow{3}{*}{ Títulos } & \multicolumn{4}{|c|}{ População } \\
\hline & \multicolumn{2}{|c|}{ urbana } & \multicolumn{2}{|c|}{ rural } \\
\hline & $n^{\circ}$ & $\%$ & $\mathrm{n}^{\circ}$ & $\%$ \\
\hline $1 / 4$ & 21 & 72,4 & 11 & 91,7 \\
\hline $1 / 8$ & 7 & 24,1 & 1 & 8,3 \\
\hline $1 / 16$ & 1 & 3,5 & - & - \\
\hline Total & 29 & 100,0 & 12 & 100,0 \\
\hline
\end{tabular}

Dos indivíduos que costumam ingerir carne de caça $24,3 \%$ (cidade) e $54,6 \%$ (rural) referem ter observado cistos hialinos em víscera de paca. Quando
Tabela 4 - Inquérito entre populacão urbana e rural do município de Sena Madureira quanto ao hábito da caça de subsistência e a ingestão de seus produtos.

\begin{tabular}{|c|c|c|c|c|}
\hline \multirow{3}{*}{ Variáveis } & \multicolumn{4}{|c|}{ População } \\
\hline & \multicolumn{2}{|c|}{ urbana } & \multicolumn{2}{|c|}{ rural } \\
\hline & $n$ & $\%$ & $\mathrm{n}$ & $\%$ \\
\hline \multicolumn{5}{|l|}{ Hábito de caçar } \\
\hline não & 617 & 77,3 & 97 & 46,0 \\
\hline $\operatorname{sim}$ & 181 & 22,7 & 114 & 54,0 \\
\hline \multicolumn{5}{|l|}{ Ingestão de carne de caça } \\
\hline não & 86 & 11,0 & 2 & 0,9 \\
\hline $\operatorname{sim}$ & 694 & 89,0 & 211 & 99,1 \\
\hline \multicolumn{5}{|l|}{ Animais } \\
\hline paca (Cuniculus paca) & 468 & 67,5 & 138 & 68,7 \\
\hline caititu (Tayassu tajacu) & 375 & 54,0 & 120 & 59,7 \\
\hline cutia(Dasyprocta aguti) & 60 & 8,6 & 25 & 11,8 \\
\hline
\end{tabular}

questionados sobre a existência destes cistos em vísceras de outros animais, o porco foi citado por 61 $(45,5 \%)$ indivíduos na cidade e $28(29,2 \%)$ na área rural (Tabela 5).

\begin{tabular}{|c|c|c|c|c|}
\hline \multirow{3}{*}{ Variáveis } & \multicolumn{4}{|c|}{ População } \\
\hline & \multicolumn{2}{|c|}{ urbana } & \multicolumn{2}{|c|}{ rural } \\
\hline & $\mathrm{n}^{\circ}$ & $\%$ & $\mathrm{n}^{\circ}$ & $\%$ \\
\hline \multicolumn{5}{|c|}{ Observação de cisto em víscera de paca } \\
\hline não & 609 & 75,7 & 94 & 45,4 \\
\hline $\operatorname{sim}$ & 196 & 24,3 & 113 & 54,6 \\
\hline Total & 805 & & 207 & \\
\hline \multicolumn{5}{|c|}{ Observação de cisto em outros animais } \\
\hline $\operatorname{sim}$ & 179 & 22,1 & 97 & 46,9 \\
\hline não & 630 & 77,9 & 110 & 53,1 \\
\hline Total & 809 & & 207 & \\
\hline \multicolumn{5}{|l|}{ Quais } \\
\hline porco & 61 & 45,5 & 28 & 29,2 \\
\hline Total & 134 & & 96 & \\
\hline
\end{tabular}

\section{DISCUSSÃO}

Por não existirem outros estudos populacionais sobre hidatidose policística, houve dificuldade em se estabelecer a validade externa dos achados. Deve-se ressaltar que as relações obtidas são discutidas utilizando como parâmetro as metanálises de relatos de casos (doentes) ${ }^{5}$.

Neste estudo a distribuição da população reagente, quanto ao sexo foi concordante com a apresentada na literatura, ou seja 1:1. Quanto à idade, diferente da literatura não parece haver nenhum grupo preponderante entre os reagentes.

A prevalência na área rural (6\%) para reação sorológica foi similar à prevalência urbana $(3,5 \%)$ considerando-se os intervalos de confiança adotados. A prevalência geral é de $4 \%$, somatória das duas áreas, e avaliados 1.064 indivíduos. Esta semelhança provavelmente se deve ao estreito contato geográfico e cultural entre os dois grupos populacionais.
Nenhum estudo que focaliza a utilização da técnica de contraimunoeletroforese (CIE) na hidatidose estabeleceu títulos de anticorpos para definir a doença ${ }^{3}$. Nem os inúmeros relatos de casos, mesmo quando utilizaram a confirmação sorológica, se debruçaram sobre a correlação clínica do valor dos títulos de anticorpos ${ }^{10112427}$.

Apesar de sua alta especificidade a técnica CIE admite a ocorrência de algumas reações cruzadas. Os parasitas principalmente os da família Taenidae e do gênero Schistosoma sp são responsáveis com maior freqüência por este fenômeno.

O ensaio de Arienti e cols, em $1997^{3}$, concluiu que as técnicas de ELISA e CIE, isoladas ou mesmo combinadas, têm um alto valor no diagnóstico das hidatidoses. 
D'Alessandro e cols, em $1979^{9}$, e Meneghelli e Martinelli, em $1991^{20}$ observaram alguns hábitos comuns no grupo de doentes, raros na população geral. A prática da caça de subsistência e o uso de seu produto como parte da dieta protéica diária foram dois deles, verificados amplamente na população de Sena Madureira.

Em entrevista realizada neste inquérito sobre hábitos locais, verificou-se a observação de cistos hialinos tanto nas vísceras das pacas abatidas como em outros animais. Destaca-se a observação destes cistos em vísceras de porcos doméstico na área urbana 45,5\% e $29,2 \%$ na rural. Não se encontrou relato sobre a possibilidade de participação deste animal no ciclo da hidatidose policística, necessitando melhor elucidação.

Seria importante a realização de um inquérito ultrasonográfico nesta população, pois cada vez mais, temse visto este método em estudos populacionais sobre hidatidose, com a vantagem de não ser invasivo, ter resultados instantâneos e apresentar custos razoavelmente discretos ${ }^{4} 123334$.

\section{REFERÊNCIAS BIBLIOGRÁFICAS}

1. Almeida SCX, Martins RLM, Moraes MAP, Viegas CA, Grillo M. Hidatidose pulmonar policística mimetizando lesões metastáticas: relato de caso. Jornal de Pneumologia 23: 261-263,1997.

2. Amaral ISA, Soares MC, Boulhosa CF Amaral EAA. Hidatidose policística por E. Vogeli llha do Marajó, Pará, BrasilAcompanhamento clínico de três anos Revista Paraense de Medicina 14: 48-53 2000.

3. Arienti HM, Guignard SI, Rinaldi DE, Elbarcha OC. Comparision of two serologic methods for the diagnosis of hydatidosis. Revista Panamericana de Salud Pública 1: 376-80,1997.

4. Carmona C, Perdomo R, Carbo A, Alvarez C, Monti J, Grauerb R, Stern D, Perera G, Lloyd S, Bazini R, Gemmell A, Yarzabal L.Risk factors associated with human cystic echinococcosis in Florida, Uruguay: Results of a mass screening study using ultrasound and serology. American Journal of Tropical Medicine and Hygiene 58: 599-605,1998.

5. D'Alessandro A. Polycistic echinococcosis in tropical America: Echinococcus vogeliand E. Oligarthrus. Acta Tropica 67: 43-65, 1997.

6. D'Alessandro A, Moraes MAP Raick AN. Polycistic hydatid disease in Brazil. Report of five new human cases and a short review of other published observations. Revista da Sociedade Brasileira de Medicina Tropical 29: 219-228,1996.

7. D'Alessandro A, Ramirez LE, Chapadeiro E, Lopes ER, and Mesquita PM. Second recorded case of human infection by Echinococcus oligarthrus. American Journal Tropical Medicine Hygiene 52: 29-33,1995.

8. D'Alessandro A, Rausch RL, Cuello C, Aristizabal N. Echinococcus vogeli in man, with a review of polycistic hydatid disease in Colombia and neighboring countries. American Journal of Tropical Medicine and Hygiene 28: 303-317,1979.

9. D'Alessandro A, Rausch RL, Morales GA, Collet S Angel D. Echinococcus Infections in Colombian Animals. American Journal Tropical Medicine Hygiene 30: 1263-1276,1981.

10. Ferreira MS, Nishioka SA, Rocha A, D'Alessandro A. Echinococcus vogeli polycistic hydatid disease:report of two brazilian cases outside the Amazon region. Transactions of the Royal Society of Tropical Medicine and Hygiene 89:286-287,1995

11. Ferreira MS, Rocha A, Gonçalves EG, Carvalho AM, Nishioka SA, Andrade NB. Relato de Caso: Um caso de hidatidose policística autóctone de Minas Gerais, Brasil. Revista da Sociedade Brasileira de Medicina Tropical 20: 181-186, 1987.

12. Frider B, Moguilensky J, Salvitti JC, Odriozola M, Cantoni G Larrieu E. Epidemiological surveillance of human hydatidosis by means of ultrasonography: Its contribution to the evaluation of control programs. Acta Tropica 79: 219-223,1999.
13. Gardner SC, Rausch RL, Camacho OCJ. Echinococcus vogeli Rausch and Bernstein,1972, from the paca, Cuniculus paca I.(Rodentia: Dasyproctidae), in the Departamento de Santa Cruz, Bolivia. Journal of Parasitology 74:399-402,1988.

14. Gottstein B, D'Alessandro A, Rausch RL. Immunodiagnosis of polycistic hydatid disease/polycystic echinococcosis due to Echinococcus vogeli. American Journal of Tropical Medicine and Hygiene 53: 558-563,1995.

15. Gottstein B, Eckert J, Fey H. Serological differentiation between Echinococcus granulosus and E. Multilocularis infection in man. Zeitschrift für Parasitekunde 69:347-356,1983.

16. Grabbe E, Kern P, Heller M. Human echinococcosis: diagnosis value of computed tomography. Tropenmedizin und Parasitologie 32: 35-38,1981

17. Iacona A, Pini C, Vicari G. Enzyme-Linked Immunosorbent Assay (ELISA) in the serodiagnosis of hydatid disease. American Journal of Tropical Medicine and Hygiene 29: 95-102, 1980.

18. Irabuena $\mathrm{O}$, Nieto $\mathrm{A}$, Ferreira AM, Battistoni $\mathrm{J}$ and Ferragut G. Characterization and optimization of bovine Echinococcus granulosus cyst fluid to be used in immunodiagnosis of hydatid disease by Elisa. Revista do Instituto de Medicina Tropical de São Paulo 422000.

19. Meneghelli UG. Calcificações hepáticas múltiplas decorrentes de doença hidática policística. Revista Goiana de Medicina 31: 53-60,1985.

20. Meneghelli UG, Barbó MLP, Magro JE, Bellucci AD, Velludo Llorach MA. Polycystic hydatid disease (Echinococcus vogeli): Clinical and radiological manifestations and treatment with albendazole of a patient from the Brazilian Amazon region. Arquivos de Gastroenterologia de São Paulo 23: 177-183,1986.

21. Meneghelli UG, Martinelli ALC. Doença hidática policística (Equinococose Neotropical). In. Castro LP, Rocha PR, Cunha AS (eds) Tópicos em Gastroenterologia Tropical MEDSI pgs 387-401,1991.

22. Meneghelli UG, Martinelli ALC, Belluci AD, Villanova MG. Polycistic hydatid disease (Echinococcus vogeli). Treatmentt with albendazole. Annals of Tropical Medicine and Parasitology 86:152-156,1992.

23. Meneghelli UG, Martinelli ALC, Llorach Velludo MAS. Cistos de Echinococcus vogeli em fígado de paca (Cuniculus paca) originária do Estado do Acre, Brasil. Revista da Sociedade Brasileira de Medicina Tropical 23: 153-155,1990.

24. Meneghelli UG, Martinelli ALC, Velludo Llorach MA, Bellucci AD, Magro JE, Barbó MLP. Polycistic hydatid disease (Echinococcus vogeli ) Clinical, laboratory and morphological findings in nine Brazilian patients. Journal of Hepatology 14: 203-210, 1992. 
25. Novak M, Kornovski B, Shimizu KY, Buist RJ. Echinococcus multilocularis- A model for imaging research. Journal of Parasitology 77: 803-805,1991.

26. O'Grady JP, Yeager CH, Esra GN a Thomas DVM. Ultrasonic evaluation of echinococcosis in four lowland gorillas. Journal of American Veterinary Medicine Association 181: 1348-1350, 1982.

27. Pacheco PRG, Komma MD, Pinto RLN, Souza LCS, Pereira LIA. Doença Hidática Policística: Relato de um caso procedente do Pará. Revista da Sociedade Brasileira de Medicina Tropical 19: 67,1986.

28. Rausch RL, Bernstein JJ. Echinococcus vogeli sp. n. (Cestoda: Taeniidae) from bush dog, Speothos venaticus (Lund). Zeitschrift für Tropenmedizin und Parasitologie 23:25-34,1972.

29. Rausch RL, D'Alessandro A. Histogenesis in the metacestode of Echinococcus vogeli and mechanism of pathogenesis in polycystic hydatid disease. Journal of Parasitology 85:410-418,1999.

30. Rausch RL, D'Alessandro A, Rausch VR. Characteristics of the larval Echinococcus vogeli Rausch and Bernstein,1972. In the natural intermediate host, the paca, Cuniculus paca
I. (Rodentia: Dasyproctidae). American Journal of Tropical Medicine and Hygiene 30:1043-1052,1981.

31. Rausch RL, D'Alessandro A, Ohbayashi M. The taxonomic status of Echinococcus cruzi Brumpt and Joyeux, 1924 (cestoda: Taeniidae) from an agouti (rodentia: dasyproctidae) in Brazil. Journal of Parasitogy 70: 295-302,1984.

32. Rodrigues-Silva R, Peixoto JRV, Oliveira RMF, Pinto R.M, Gomes DC. An Autochthonous Case of Echinococcus vogeli Rausch \& Bernstein, 1972 Polycystic Echinococcosis in State of Rondônia, Brazil. Memórias do Instituto Osvaldo Cruz 97:123-126,2002.

33. Shambesh MA, Graig PS, Macpherson CNL, Rogan MT, Gusbi AM \& Echtuish FE. An extensive ultrasound and serologic study to investigate the prevalence of human cystic echinococcosis in Northern Lybia. American Journal of Tropical Medicine and Hygiene 60: 462-468,1999.

34. Soares MCP, Amaral ISA. Images in hepatology. Polycystic Echinococcosis by E.vogeli in the Amazon Region. Journal of Hepatology 28:908,1998. 